\title{
The Shuffle Motor: A High Force, High Precision Linear Electrostatic Stepper Motor
}

\author{
Niels Tas*, Jeroen Wissink*, Louis Sander**, Theo Lammerink* and Miko Elwenspoek* \\ *MESA Research Institute, University of Twente, P.O. Box 217, 7500 AE Enschede, the Netherlands. (N.R.Tas@el.utwente.nl) \\ ** Philips Research, WA 1-2-10, Prof. Holstlaan 4, 5656 AA Eindhoven, the Netherlands
}

\section{SUMMARY}

The shuffle motor is a electrostatic stepper motor that employs a mechanical transformation to obtain high forces and small steps. A model has been made to calculate the driving voltage, step size and maximum load to pull as well as the optimal geometry. Tests results are an effective step size of about 85 $\mathrm{nm}$ and a maximum produced force of about $40 \mu \mathrm{N}$ at $40 \mathrm{~V}$ clamping voltage and $25 \mathrm{~V}$ actuator voltage.

Keywords: Electrostatic motor, linear motor, stepper motor, surface micromachining.

\section{INTRODUCTION}

Linear motors that apply a walking motion are able to add small steps in order to obtain large translations [1-5]. The possibility to combine a high resolution with a large total stroke makes them attractive for applications like high density data storage or scanning microscopy. The electrostatic stepper motor presented here is fabricated in a multi level polysilicon surface micromachining process. The main feature of the shuffle motor is the use of a mechanical transformation to increase the relative weak electrostatic forces, and to obtain small steps needed for high precision positioning [3].

\section{PRINCIPLE OF OPERATION}

Figure 1 shows the principle of the shuffle cycle. First the front is clamped and the actuator plate is deflected downward. This causes the back to shift forward. Next the back is clamped, the front and the actuator plate are released. This causes the front to shift forward. The electrostatic normal force acting on the actuator plate induces a friction force in the feet of about the same magnitude. Due to the built-in mechanical transformation, the developed lateral force will be

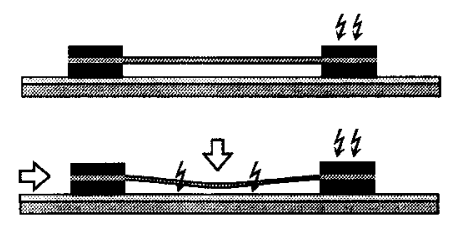

Figure 1: Principle of operation. First the front is clamped and the actuator plate is activated. This causes the back to shift forward. Next the back takes over the clamping,

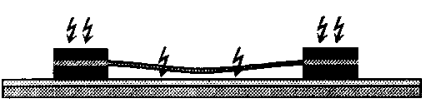
the front is released and the actuator plate is released. This causes the front to shift forward. larger than the friction force and is high enough to make the feet sliding. The deformation of the plate by the electrostatic forces lead to a small but forceful contraction of the plate. The force of contraction can be estimated by concentrating the electrostatic force in the center, neglecting the bending stiffness of the plate and making a force balance for the electrostatic force $F_{\mathrm{e}}$ and the tensile force $S$ (figure 2). The shape of the deformed plate is approximated by a triangle and the center deflection $y$ is assumed much smaller than the plate length $2 l$.

$S=\frac{1}{2} \cdot \frac{l}{y} \cdot F_{e}$

Typical values are $l=100 \mu \mathrm{m}$ and $y=0.2 \mu \mathrm{m}$, which gives a changing transformation ratio from $\infty$ to 25 .

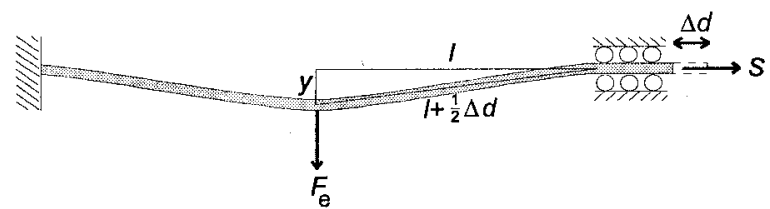

Figure 2: Force balance of the actuator plate. A tensile force $S=l \cdot F_{e} / 2 y$ is developed, if the bending stiffness of the plate can be neglected. Based on the triangle approximation, a step of $\Delta d=y^{2} / l$ is calculated.

A step size $\Delta d=y^{2} / l$ can easily be calculated using the triangular deflection profile, assuming small values of $y / l$ [3]. For a center deflection of $2 \mu \mathrm{m}$ and a plate length of $200 \mu \mathrm{m}$ this results in a step size of about $40 \mathrm{~nm}$. A thin plate is needed in order to keep the bending stiffness and thereby the driving voltage low. However, a thick plate is needed in order to keep the tensile strain in the plate low. A more detailed model has been made that enables us to find the optimum plate thickness.

\section{MODEL OF THE TRANSDUCER}

The actuator plate can be seen as a buffer element with two power ports. The external behavior of the transducer can be described by four variables (figure 3 ). A model has been made that expresses developed stroke $\Delta d$ and stored charge $Q$ as a function of the tensile load force $S$ and voltage $U$.

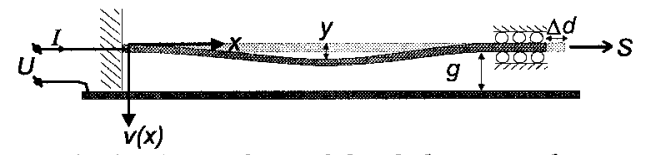

Figure 3: Ideal Physical Model of the transducer. $x$ is the position along the plate, $y$ is the center deflection, $v(x)$ is the deflection of the plate. 
$3 \mathrm{~A} 2.02$

The deflection of the actuator plate is described by a fourth order non linear differential equation. We have not been able to solve this equation. A solution has been obtained for the case where the electrostatic force is concentrated in the center of the plate (like in figure 2), and the case where the electrostatic force is taken uniformly along the plate length. We introduce the model for the central force case. The deflection $v(x)$ of the plate which is under a tensile force $S$, as a function of the central normal force $F_{e}$ is given by:

$$
\begin{aligned}
& v\left(F_{e}, K, z\right)= \\
& =\frac{F_{e} l^{3}}{E I} \frac{1-\cosh (K)-\cosh (K z)+z K \sinh (K)+\cosh (K(z-1))}{2 K^{3} \sinh (K)}
\end{aligned}
$$

The distance $x$ is normalized using half the plate length $l, z=x / l$ $(0<z<1)$. The tensile force $S$ is present in parameter $K$ which is defined as:

$$
K \equiv l \cdot \sqrt{\frac{S}{E I}}
$$

The parameter $K$ determines if the transducer is bending dominated or tensile force dominated. The deflection function can be split in an amplitude function $y\left(F_{e}, K\right)$ and a shape function $V(K, z)$. The amplitude function expresses the center deflection $y$.

$v(K, z)=y\left(F_{e}, K\right) \cdot V(K, z)$

The shape function is plotted in figure 4 a for a range of $K$. The center deflection $y\left(F_{\mathrm{e}}, K\right)$ depends linearly on $F_{e}$. We can therefore describe the center deflection as a function of $F_{e}$ as a stiffness function $k(K)=F_{e} / y$ :

$$
k(K)=\frac{24 E I}{l^{3}} \cdot\left\{\frac{1}{3} \frac{\left(\frac{1}{2} K\right)^{3}}{\left(\frac{1}{2} K\right)-\tanh \left(\frac{1}{2} K\right)}\right\} \equiv \frac{24 E I}{l^{3}} G(K)
$$

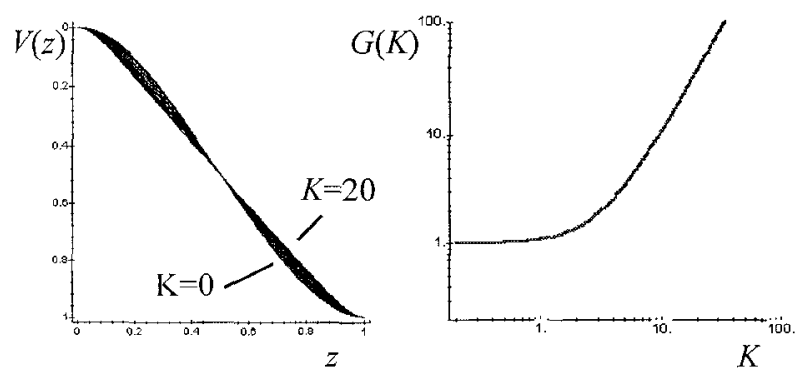

Figure 4a) The shape function $V$ versus $z$ for various $K$ in a linear plot. b) The stiffness factor $G(K)$.

In Eq. (5) $G(K)$ is the stiffness factor, that is a measure for the stiffening due to the tensile force $S$. Figure $4 \mathrm{~b}$ shows the stiffness factor $G(K)$. For small $K$ the effective stiffness is bending dominated and is consistent with the formulas for pure bending [6]:
$k_{b}=\frac{F_{e}}{y}=24 \frac{E I}{l^{3}} \quad(K<<\sqrt{12})$

For large $K$ the tensile force acts as an effective stiffness $F_{e} / y$, which is consistent with Eq. (1): The normal force $F_{\mathrm{e}}$ increases linearly with the center deflection $y$.

$k_{s}=\frac{F_{e}}{y}=\frac{2 \cdot E I \cdot K^{2}}{l^{3}}=\frac{2 S}{l} \quad(K \gg>\sqrt{ } 12)$

The turnover point is where the stiffness ratio $k_{\mathrm{s}} / k_{\mathrm{b}}=1$. From Eq. (6) and (7) it follows that this is at $K^{2}=12$. Using the stiffness function $k(K)$ an expression for the pull-in voltage as a function of $K$ can be derived:

$U_{p i}=\sqrt{G(K)} \sqrt{0.8 \frac{E \cdot t^{3} \cdot g^{3}}{\varepsilon \cdot l^{4}}}$

Where $t$ is the thickness of the plate, and $\varepsilon$ is the permittivity. The factor 0.8 is a truncated value: it increases from 0.75 for $K$ $=0$ to 0.83 for $K=\infty$. The step size can be found by calculating the path length along the plate using the deflection function $v(x)$. For small slopes $\mathrm{d} v / \mathrm{d} x$ the step size is given by:

$\Delta d=2 \int_{0}^{l} \sqrt{\left(1+\left(\frac{d v}{d x}\right)^{2}\right)} d x-2 l$

The central force shape function $v$ approximates the triangle shape for large $K$. For small $K$ the curves of the deformed plate are smoother and the path length is slightly larger:

$$
\begin{array}{cl}
\Delta d=\frac{6}{5} \frac{y^{2}}{l} & K \ll \sqrt{ } 12 \\
\Delta d=\frac{y^{2}}{l} & K \gg \sqrt{ } 12
\end{array}
$$

The lateral strain $\Delta s$ in the plate for a given load $S$ is found using the lateral plate stiffness. The developed step is completely lost if $\Delta s=\Delta d$. Taking $\Delta d$ from Eq.(10b) and $y=g$ we find a maximum allowable $K$ for which the step is just completely lost due to lateral strain:

$$
K_{\max }=\frac{g}{t} \sqrt{6}
$$

\section{DESIGN \& REALIZATION}

The moving part of the motor consists of two polysilicon layers. A thin polysilicon for the actuator plate, and a thick polysilicon for the frame in which the plate is hung. The thick polysilicon for the frame is needed to give the frame the desired stiffness properties. The frame consists of a front part and a back part, connected by the stretching springs and by the plate. Both the front and the back part contain two clamp feet (figure 5). Anti-sticking bumps have been made all along the clamp feet. Polysilicon electrodes have been made underneath the feet and the plate, so that the front feet, the back feet and the plate can be activated independently. The moving part is 
grounded during operation. The frame is contracted by deflecting the plate downward (figure 6), and stretches by means of the stretching spring. The stiffness of the four stretching springs together is about $12 \mu \mathrm{N} / 10 \mathrm{~nm}$. The whole moving part is supported by two folded springs which serve as a linear guidance with a stiffness of $1 \mu \mathrm{N} / \mu \mathrm{m}$. The folded springs are also used to ground the moving part.

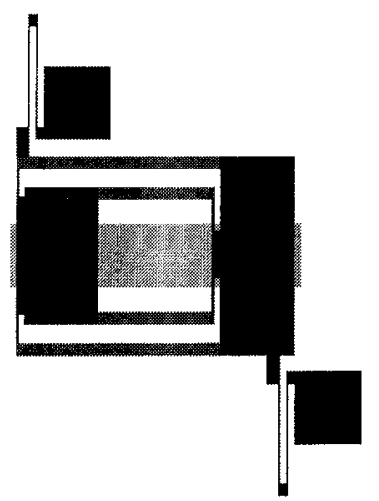

Figure 5: The frame consists of two parts, a high part (dark gray) and a low part (medium gray). The low part consists of the four clamp feet. The high part consists of the folded support springs and the large stiff parts that connect the clamp feet and the actuator plate (light gray). The support springs are anchored at the black squares.

The actuator has been designed to carry a load $S=0.1 \mathrm{mN}$ at a driving voltage below $50 \mathrm{~V}$. The thickness $t$ of the plate is chosen $0.5 \mu \mathrm{m}$, the width $w=100 \mu \mathrm{m}$ and the length $2 l=200$ $\mu \mathrm{m}$. With this plate length a step size of $40 \mathrm{~nm}$ is made if we choose $g=2 \mu \mathrm{m}$. Using a Young's modulus $E=150 \mathrm{GPa}$ this gives a stiffness parameter $K=2.6$ and a stiffness factor $G(K)$ $=1.7$. Using Eq. (8) a pull-in voltage of $14.7 \mathrm{~V}$ can be calculated for the motor loaded by $0.1 \mathrm{mN}$, well below $50 \mathrm{~V}$. For this choice of $t$ and $g$ we find $K_{\max }=10$. At $0.1 \mathrm{mN}$ load the parameter $K=2.7$ is well below this value, so only a small part of the step is lost due to lateral strain.
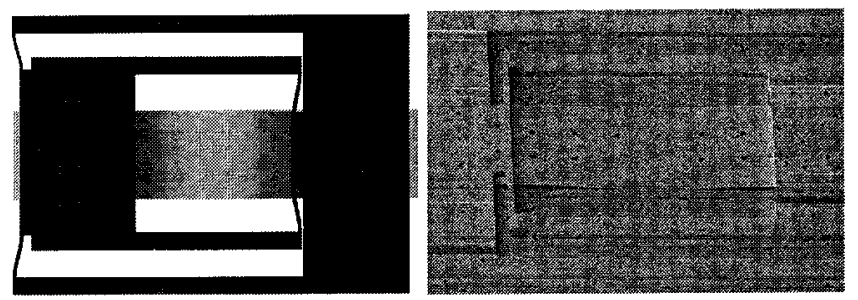

Figure 6: (left) Deformation of the stretch springs due to contraction of the plate, (right) SEM picture of a motor with the actuator plate deflected downward.
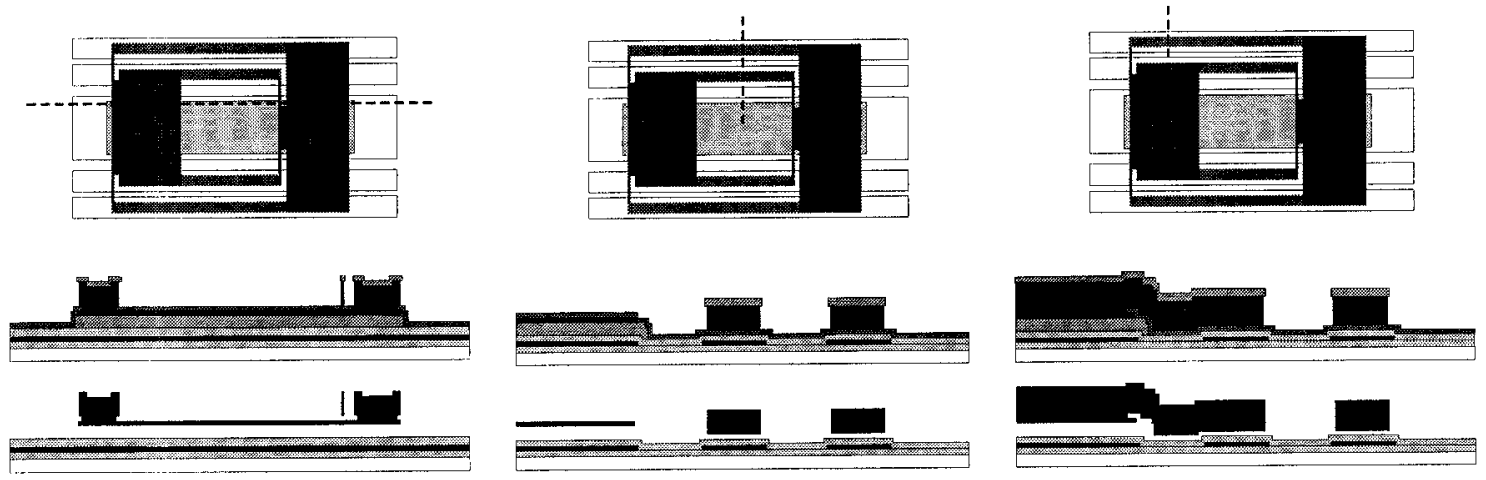

\section{Fabrication process}

On a $3 "$ substrate wafer a $1.0 \mu \mathrm{m}$ low stress LPCVD silicon nitride layer is grown to insulate the ground electrodes from the substrate wafer. Next a $0.5 \mu \mathrm{m}$ LPVCD polysilicon layer is deposited, doped with Boron by solid source indiffusion and patterned using RIE to form the electrodes. A second low stress LPCVD silicon nitride layer of $0.5 \mu \mathrm{m}$ is grown to cover the electrodes. In this nitride layer the contact pads and anchor holes are etched using RIE. On top of the nitride insulation layer the first sacrificial oxide layer $(2.4 \mu \mathrm{m})$ is deposited using PECVD to create the gap between the actuator plate and ground electrode. The next layer $0.5 \mu \mathrm{m}$ of LPVCD polysilicon doped with boron, to form the actuator plate. After the patterning of the actuator plate (RIE) the first sacrificial layer is patterned (RIE). Only where the high parts in the frame are made (figure 5), the oxide is kept. Next a $0.5 \mu \mathrm{m}$ second sacrificial layer is grown, using TEOS instead of PECVD to obtain better step coverage. The holes to form the anti sticking bumps (150 nm deep) and the plate-frame contact holes are etched in HF. Next, a $4.0 \mu \mathrm{m}$ third LPCVD polysilicon layer is deposited and doped, which after patterning in RIE forms the frame. Last step is removing both sacrificial layers using $50 \%$ HF and freeze drying to avoid sticking of the free hanging structures. Holes have been etched in both the plate and the frame at intervals of $30 \mu \mathrm{m}$ in order to shorten the sacrificial oxide etch time. In figure 8 three cross sections are shown just before and after the sacrificial layer etch. Figure 7 shows a close up around one of the two inner stretch springs.

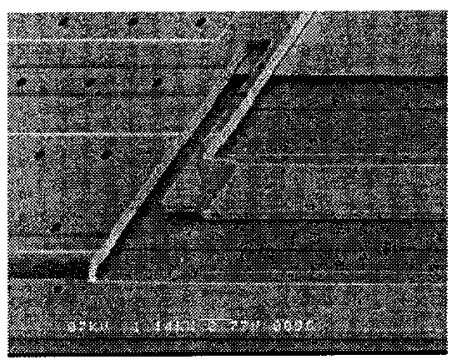

Figure 7: Close up of one of the two inner stretch springs, showing the different levels in the motor.

Figure 8: Cross sections just before and after the sacrificial oxide etching. 


\section{TEST RESULTS}

The measured zero load pull-in voltage varied between 12 and $13 \mathrm{~V}$. This is reasonable close to the $11 \mathrm{~V}$ predicted by the model. As can be seen in figure 10, the deflection profile after pull-in is different from the assumed profile in our model. Based on the profile in figure 10 the expected step size is about $140 \mathrm{~nm}$ at $25 \mathrm{~V}$ actuation voltage. Repeated pull-in showed stiction of the actuator plate to the bottom electrode. The stiction was induced by charging of the insulating nitride covering the under electrode. The charging could be greatly reduced by applying a modulated AC voltage (figure 9). The tests have been done using a $25 \mathrm{kHz}$ block wave. The transition time in the block signal is below the mechanical response time of the plate. Therefore, the $\mathrm{AC}$ voltage results in the same deflection of the plate as a DC voltage with the same amplitude.

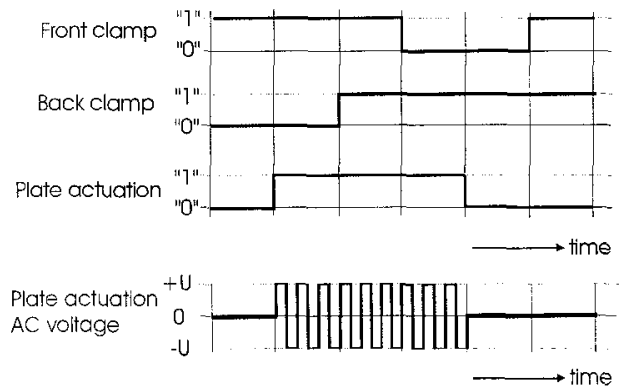

Figure 9: (Top) The control signals for a complete walking cycle, (Bottom) The modulated AC-voltage for the plate actuation channel.

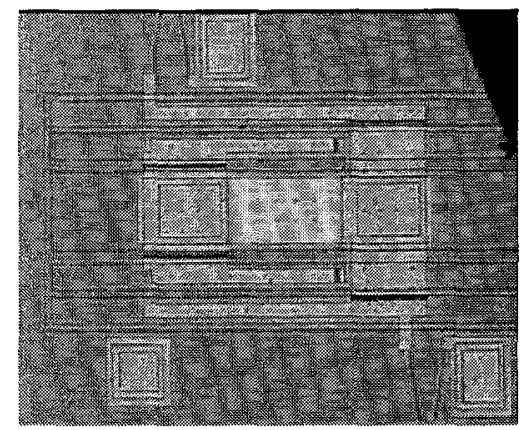

Figure 10: Microscope picture of the motor at full reach. The activated plate is pulled flat against the bottom electrode.

Walking experiments have been done using the modulated ACvoltage control. The maximum obtained reach was $43 \mu \mathrm{m}$ (figure 10) at a plate actuation voltage of $25 \mathrm{~V}$ and a clamp voltage of $40 \mathrm{~V}$ and a stepping frequency of $1160 \mathrm{~Hz}$. Based on the stiffness of the support springs, this corresponds with an effective generated force of $43 \pm 13 \mu \mathrm{N}$. At $25 \mathrm{~V}$ the actuator should be able to produce a force up to $0.4 \mathrm{mN}\left(K=K_{\max }\right)$. Slip in the clamps is the most probable explanation for the lower than expected produced force. Considerable slip occurs when the AC-driving voltage is used. The clamps are shortly released when the voltage switches from positive to negative. During normal operation there is probably a dynamic equilibrium between walking forward and slipping backward. The speed of the motor has been measured at a function of the cycle frequency (figure 11). The highest measured speed of the motor is $100 \pm 10 \mu \mathrm{m} / \mathrm{s}$, at a cycle frequency of $1160 \mathrm{~Hz}$. This frequency was the highest that could be produced with in our set up. Higher cycle frequencies should be possible, because the resonance frequency of the actuator plate is about $60 \mathrm{kHz}$. The measured speeds could be reproduced with a maximum deviation of $25 \%$ between three different motors. The slope of the graph is a measure for the effective step size, which is $85 \pm$ $9 \mathrm{~nm}$. The smaller than expected effective step size can be explained by the considerable slip in the clamps due to the used AC-driving voltage.

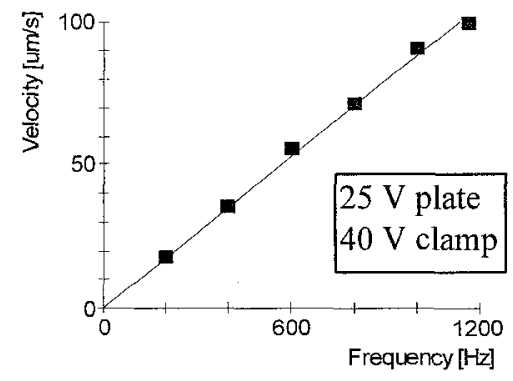

Figure 11: Measured velocity as a function of the cycle frequency). The inaccuracy in the velocity is smaller than $10 \%$

\section{CONCLUSIONS}

This paper shows the feasibility of the use of a mechanical transformation in order to increase both the force and the resolution of an electrostatic linear stepper motor. A force of about $43 \mu \mathrm{N}$ has been measured at an applied actuator voltage of $25 \mathrm{~V}$ and a clamp voltage of $40 \mathrm{~V}$. The measured step size equals $85 \pm 9 \mathrm{~nm}$. The force produced by the motor is limited by the friction in the clamps. Slip in the clamps is caused by the AC-voltage actuation of the clamps. The highest measured speed of the motor is $100 \pm 10 \mu \mathrm{m} / \mathrm{s}$, at a cycle frequency of $1160 \mathrm{~Hz}$. Electrostatic charging of the insulating silicon nitride has been observed. The charging could be diminished by a modulated AC-voltage actuation.

\section{ACKNOWLEDGMENTS}

This research is carried out in corporation with Philips Research. The authors would like to thank Meint de Boer and Erwin Berenschot for their help with and advises on the processing. We are thankful to Arie Kooij for making the masks, and Bert Otter for making the SEM-photographs.

\section{REFERENCES}

[1] Akiyama et al., J. MEMS., vol. 2-3, 1993, pp. 106-110.

[2] M.P. Koster, Proc. Actuator 94, June 1994, pp. 144-148.

[3] N.R. Tas et al., Proc. MME 95, Copenhagen, pp. 128-131.

[4] R. Yeh et al., Proc. Transducers '95, Vol. 2, pp. 346-349.

[5] R. Yeh et al., DSC-Vol. 55-2, ASME MEMS 94, pp. 747-754.

[6] Gere J.M., Timoshenko S.P., Mechanics of Materials, Chapman \& Hall, London, 1991. 